\title{
Intraoperative Venous Injury, CTCAE
}

National Cancer Institute

\section{Source}

National Cancer Institute. Intraoperative Venous Injury, CT CAE. NCI Thesaurus. Code C143614.

A finding of damage to a vein during a surgical procedure. 